\title{
ARTICLE
}

\section{Joint Application of Perl Scripts and MCNPX in Solving the Dynamic-Geometry Related Problems in Proton Beam Radiotherapy}

\author{
Fada GUAN *, John W. POSTON, Sr. and Leslie A. BRABY \\ Department of Nuclear Engineering, Texas A\&M University, College Station, TX, 77843-3133, USA
}

\begin{abstract}
The Monte Carlo code MCNPX has been verified to be a suitable tool in predicting dose distributions in proton therapy problems. In the passive-scattering treatment technique, a range modulation wheel rotating at a fixed rate in the treatment process is used to generate a spread-out Bragg peak depth-dose distribution. This dynamic geometry cannot be modeled in the simulation of MCNPX. In this study, a so-called "replacing dynamic by static" method was adopted to solve this problem. This method was implemented by a script_- "mcnp_pstudy," written in Perl programming language, which was used to set up a series of MCNPX input files, each of which contains the parameters of a specified step of the wheel, and invoke MCNPX to simulate each problem successively. The other Perl script_- "merge_mctal" was used to merge all the separate simulation results into one file containing the simulation results that are equivalent to a "dynamic" simulation. The simulation results such as particle fluence and dose distributions were obtained from this study. This study provided an example of joint application of Perl scripts and MCNPX.
\end{abstract}

KEYWORDS: proton, radiotherapy, MCNPX, Perl, mcnp_pstudy, merge_mctal

\section{Introduction}

Proton therapy was first proposed as a treatment modality for cancer therapy by Robert Wilson in 1946. ${ }^{1)}$ The accurate dose delivery ability of protons makes it possible to be used in clinical application. Most of the current proton therapy facility units are mounted with a passive-scattering nozzle in which a rotating range modulation wheel is used to produce the spread-out Bragg peak (SOBP) depth-dose distribution in the target tumor during the treatment. ${ }^{2)}$ This dynamic geometry adds additional difficulty and complexity for the Monte Carlo dose calculations, especially when using the static Monte Carlo simulation codes. The dynamic beam scanning technique also meets a similar problem when using the Monte Carlo simulation to obtain the real-time dose distribution. Paganetti has provided a solution to obtain the dose distribution produced by the time-dependent geometries by using the Monte Carlo code Geant4.,3)

MCNPX is a general-purpose Monte Carlo particle transport code, and it has been widely used in the validation of a proton treatment nozzle and in predicting the dose distribution from clinical proton beams. ${ }^{4-6)}$ In this study, we used the latest public release of this code-MCNPX version 2.6.0 to simulate the transport and interaction of protons with matter. Currently, one limitation of the application of MCNPX is that it cannot be used to simulate the problem with dynamic geometries and variable settings in a single model. This study will provide a method to obtain the simulation results equivalent to a "dynamic" simulation. This method can significantly save time in preparing the simulation input files and post-processing the simulation results.

*Corresponding author, E-mail: guanfada@tamu.edu

(C) 2011 Atomic Energy Society of Japan, All Rights Reserved.

\section{Materials and Methods}

\section{Design of a Double-Scattering System and a Range Modulation Wheel}

In a passive-scattering treatment nozzle, the incident narrow proton beam is broadened laterally by a double-scattering system, and meanwhile the beam is modulated to be a SOBP beam longitudinally by a rotating range modulation wheel. These two components in the nozzle play key roles in forming a clinical proton beam. In this study, these two components were designed by using a specialized computer program-NEU. ${ }^{7)}$ The design process of this program is based on multiple Coulomb scattering theory and experimental pristine Bragg peak depth-dose distributions. ${ }^{6,7)}$

The first scatterer is usually a flat metal foil. According to the multiple scattering theory, the particle fluence after the first scatterer is not distributed uniformly laterally but is best represented by a Gaussian distribution. ${ }^{8}$ Thus the dose distribution laterally is also in a Gaussian-curve shape. Particles in the central beam need more scattering to produce a uniform fluence laterally. A Gaussian-curve shaped scatterer is added to the beam line to achieve this goal. The current design is to integrate the range modulation wheel into the double-scattering system to constitute a beam scattering-and-modulating unit. ${ }^{7)}$ The modulation parts are usually mated on the first-scatterer parts, and they are combined to be a "wheel" composed of several steps. The benefits of this approach are two-fold: saving space and decreasing scattering near the patient. This wheel is called "S1" in the NEU code. The second scatterer is a Gaussian-curve shaped structure apart from S1, and it is called "S2" in the NEU code. 


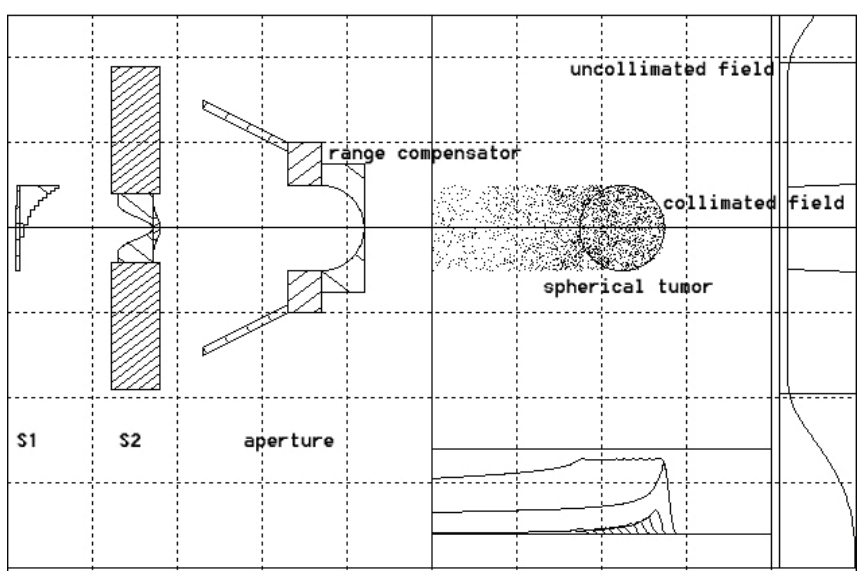

Fig. 1 The illustration of NEU design results. The size shown does not reveal the real geometry size. A hypothetical spherical tumor exists in water. Only the planar dose map (dots density stands for the relative dose), depth-dose along central axis and transverse dose across mid-SOBP are depicted, but the tracks of particles are not shown. The initial beam energy is $250 \mathrm{MeV}$; the expected SOBP width is $10 \mathrm{~cm}$; the SSD is $3 \mathrm{~m}$; the uncollimated beam-field radius is $17.7 \mathrm{~cm}$; the collimated radius is $5 \mathrm{~cm} .{ }^{9,10)}$ The calculated number of steps in $\mathrm{S} 1$ is eleven in this example.

Both of S1 and S2 are bi-material components. The reference planes of S1 and S2 are also the mating surfaces of the two different materials. In S1, on the up-stream side of the mating surface are the scatterers made of high- $Z$ material, e.g., lead, and on the down-stream side are the range modulation parts made of low-Z material, e.g., Lexan (plastic). In practice, the angle fraction (or relative width) of each step in $\mathrm{S} 1$ stands for the weighting factor of the pristine Bragg peak depth-dose distribution from that step in forming the SOBP dose curve. In S2, on the up-stream side is the inner-contoured compensation part made of low-Z material, e.g., Lexan, and on the down-stream side is the contoured scatterer made of high- $Z$ material, e.g., lead. In this study, Lexan $\left(\rho=1.2 \mathrm{~g} \mathrm{~cm}^{-3}\right)$ and lead $\left(\rho=11.35 \mathrm{~g} \mathrm{~cm}^{-3}\right)$ are used as the materials in a NEU design.

The NEU code can be used to calculate the number of steps in S1 and the thickness and weighting factor of each step and the geometry parameters of S2 according to the design requirements, such as the energy of initial beam, the source surface distance (SSD), the pristine Bragg peak dose curve for initial beam (Due to the lack of experimental data, a dose curve from MCNPX simulation was used instead of the experimental curve.), the expected beam-field size and SOBP width, etc. The results of one NEU design example used in this study are shown in Fig. 1.

\section{Monte Carlo Model of a Simplified Nozzle in MCNPX}

The key point of this study was not to build a real treatment nozzle in detail but only to provide a method to obtain dynamic simulation results. Hence, the Monte Carlo model used in this study was oversimplified compared with a real clinical treatment nozzle. For instance, we used an ideal point source emitting mono-energetic and mono-directional protons instead of the real pencil beam whose energy spectrum and initial angular-spatial distribution are both in Gaussian distribution.

The geometry and material settings of the double-scattering system and range modulation wheel adopted the design results from the NEU program. Other parameters, such as SSD and field size were consistent with the settings in the NEU design process. Other components, such as collimators, range shifters and compensators were not provided in this model. The direction of the initial $250 \mathrm{MeV}$ pencil beam was set as $\mathrm{x}$-axis (the central axis for this model). A water box with the size of $40 \mathrm{~cm} \times 80 \mathrm{~cm} \times 80 \mathrm{~cm}$ was used to score the desired quantities, such as particle current, dose and particle fluence. The front surface of the cubic water box is perpendicular to $\mathrm{x}$-axis.

For physics settings, neutrons and recoil light ions were inhibited for the current study to see the results contributed by only protons. Vavilov model was chosen as the charged-particle straggling model. ${ }^{11)}$ CEM03 (Cascade-Exciton Model 03) model was chosen as the physics model to deal with the nuclear reactions. ${ }^{12)}$

A rectangular mesh tally was used to obtain the "cell-wise" fluence and dose distributions in the water phantom. The F1 tally with energy card was used to obtain the kinetic energy spectrum of the protons incident on the front surface of the water phantom. Considering the computation time and precision requirements of the results, the size of each scoring cell is set to $0.5 \mathrm{~cm} \times 1 \mathrm{~cm} \times 1 \mathrm{~cm}$ in the water phantom. The NPS (number of particles) is set to ten million for each run to ensure the tally results to meet the requirement of $10 \%$ uncertainty in statistics. ${ }^{13)}$

\section{Replacing Dynamic by Static}

So far, we have known the materials and positions of the components used in the MCNPX simulation, but we cannot make the range modulation wheel rotate during the simulation like a real clinical treatment. Since parameters for each step are known from the NEU design, we can set up a series of MCNPX input files, in each of which, only one step is used and the weighting factor of each step is used as the initial "weight" of the source particles in the Monte Carlo simulation. After executing each simulation, the results from different cases are summed. This sum is the same as the result from a rotating wheel. We call it "replacing dynamic by static" method. However, the number of steps in a wheel is usually more than ten, so the time required in preparing the MCNPX input files and collecting the simulation results is large. If some specialized computer programs or scripts can be applied to aid the preparation and collection, more time can be saved.

One script named "mcnp_pstudy" written in Perl computer programming language was used to implement the "replacing dynamic by static" method in this study. It was originally developed to aid the MCNP5 simulation. ${ }^{13,14)}$ In this study, we modified some parameters of this script and applied it in the MCNPX simulation. 
For a certain nozzle model, only one input file written in the MCNPX format was required to prepare, which included "symbolic" variables and real values for the "mcnp_pstudy" script. We defined the thickness and weighting factor of each step in S1 as the "symbolic" variables in this "monp_pstudy" input file. The execution process of "monp_pstudy" includes three stages: setting up input files, invoking MCNPX simulation and collecting simulation results. In the first stage, a series of case directories are created and the real values are assigned to the symbolic parameters to produce one real MCNPX input file named "inp" in each case directory. The number of cases was equal to the number of steps in S1. In the second stage, the MCNPX code was invoked to execute all the "inp" files successively and after each run, one output file named "mctal" was created in the corresponding case directory. The current "monp_pstudy" script does not support the data collection for MCNPX mesh tally results because the format of output file for MCNPX is different from the one from MCNP5. The task of data collection was completed by using a different Perl script.

\section{Combination of the Simulation Results}

The other Perl script used to merge the simulation results from different cases is called "merge mctal."15) It was also originally developed to aid the MCNP5 simulation, and it cannot directly be used to combine data from MCNPX simulation. The method we used in this study was modifying the output files "mctal" from MCNPX format to MCNP5 format. The main modification included changing the source particle types and changing the mesh tally to F5 tally. After the execution of "merge_mctal," a new "mctal" file containing the combined data is produced. To make this new "mctal" file able to be read in by MCNPX, it must be changed back to MCNPX format.

\section{Results and Discussion}

\section{One-Dimensional Dose and Fluence Distributions}

The SOBP curve and the pristine depth-dose distributions along the central axis in the water phantom are shown in Fig. 2. The depth-fluence distributions along the central axis are shown in Fig. 3. The shape of the SOBP curve confirms that the "replacing dynamic by static" method is suitable to obtain the dose distribution of a near-clinical proton beam. The particle fluence decreases with depth increasing as expected. The depth-dose distribution and transverse-dose distributions at three depths on SOBP are shown in Fig. 4. The transverse-dose distributions show that the penumbrae are large for scattered protons, which can be reduced by applying beam collimators in the nozzle. In the transverse dose distribution plots, the dose line is not as flat as expected, with the outer dose higher than inner. One of the reasons for this is that the scattering system over-scattered the initial beam outside slightly.

The comparison of SOBP curves between NEU design and MCNPX simulation is not provided because the size of each dose-scoring cell is not provided explicitly in NEU and the mechanism of dose calculation in NEU is different from the one in MCNPX.

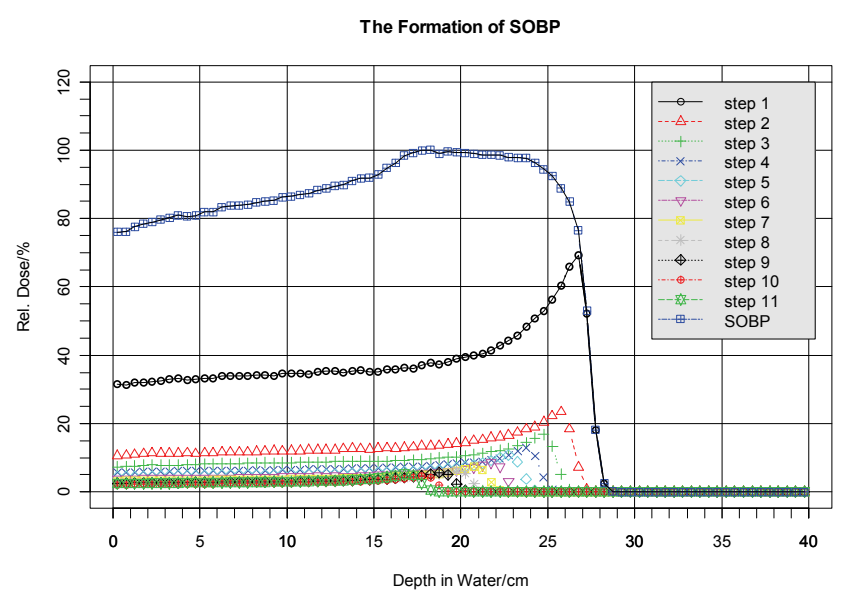

Fig. 2 The formation of SOBP by weighted depth-dose curves from different steps.

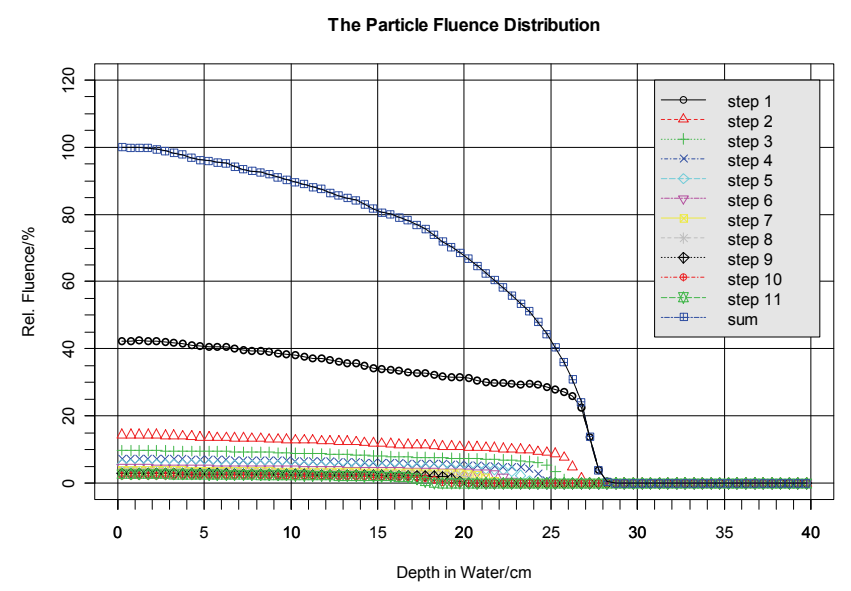

Fig. 3 The percent depth-fluence distribution in the water phantom along x-axis.
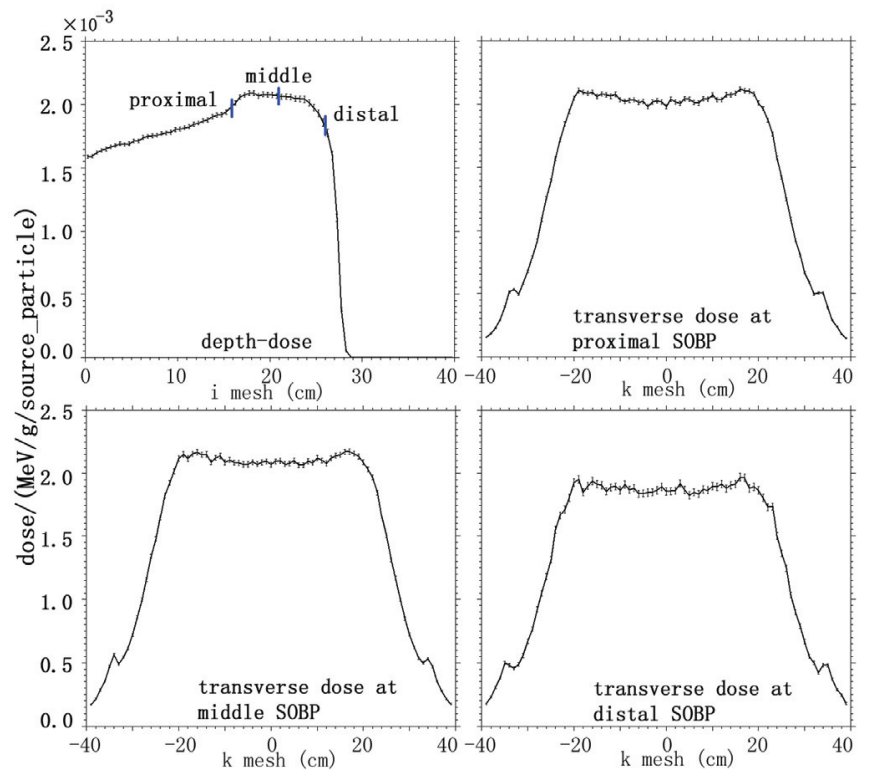

Fig. 4 The depth-dose distribution along central axis and transverse-dose distributions at three depths on the SOBP curve. The unit of absorbed dose is normalized to $\mathrm{MeV} \mathrm{g}^{-1}$ per source particle. The i-mesh lies in the $\mathrm{x}$-direction and the k-mesh lies in the z-direction. The error bar stands for the abstract statistic error in the MCNPX simulation. 


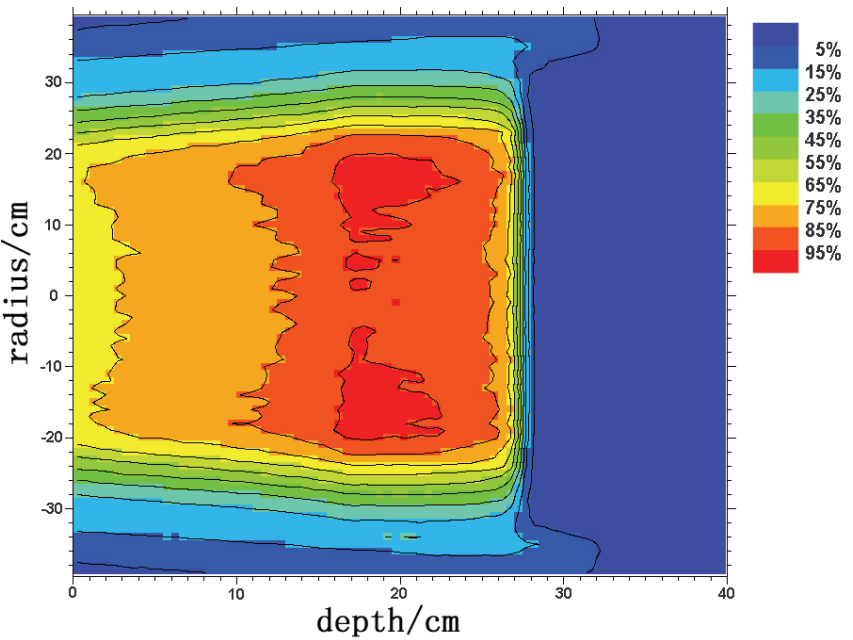

Fig. 5 The contour dose distribution in the central layer ( $x-z$ plane, y: $-0.5 \sim 0.5 \mathrm{~cm}$ ). The absorbed dose values in percent are shown in different colors.

\section{Two-Dimensional Dose and Fluence Distributions}

The contour dose and fluence distributions in the central layer (y: $-0.5 \sim 0.5 \mathrm{~cm}$ ) are shown in Figs. 5 and $\mathbf{6}$. These figures clearly show that the initial narrow beam has been broadened to be a broad beam with the expected field size.

\section{Kinetic Energy Spectrum of the Broad Beam}

The kinetic energy spectrum of the broad beam entering the water phantom is shown in Fig. 7, from which it can be seen that the kinetic energies of most particles are between $150 \mathrm{MeV}$ and $230 \mathrm{MeV}$. The energy spectrum also verifies that the SOBP dose curve is formed by a "spread-out" energetic beam produced by the scattering-and-modulation system.

\section{Conclusion}

In this study, the application of "monp_pstudy" script successfully solved the problem that MCNPX cannot be used to simulate transport problems with variable parameters and dynamic geometries. If some parameters in the output files were modified first, the "merge_mctal" script can be used to deal with the output files produced by MCNPX. The application of these two Perl scripts provided an effective method for the Monte Carlo simulation of a simplified MCNPX-based proton-therapy treatment nozzle model.

However, this study was still in the preliminary stage, and more complex simulation parameters would be set to make the model much closer to a real clinical treatment nozzle and the simulation results would be compared with the experimental results in the future study.

\section{Acknowledgment}

We thank the author of the NEU code, Dr. Bernard Gottschalk at Harvard University, USA, and all the members in MCNP and MCNPX development group at Los Alamos National Laboratory, USA. We thank the researchers at M.D.

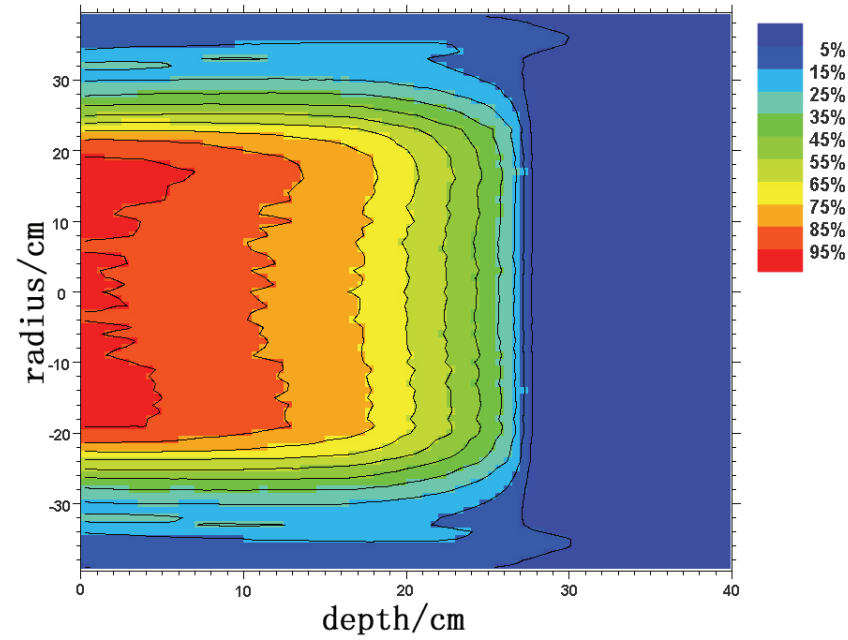

Fig. 6 The contour fluence distribution in the central layer $(\mathrm{x}-\mathrm{z}$ plane, $\mathrm{y}:-0.5 \sim 0.5 \mathrm{~cm})$. The particle fluence values in percent are shown in different colors.

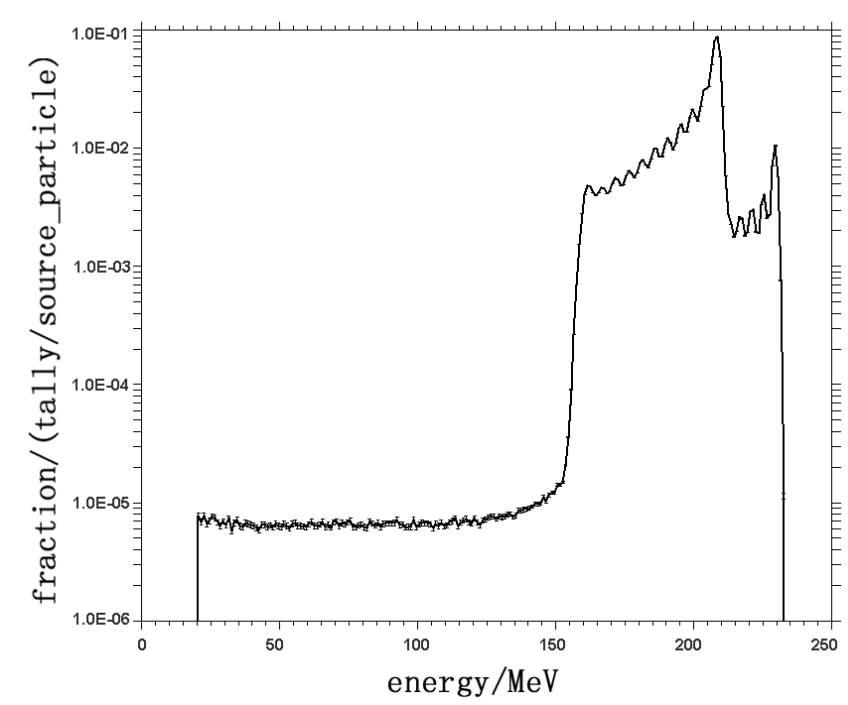

Fig. 7 The kinetic energy spectrum of protons incident on the front surface of the water phantom. The unit is normalized to tally per source particle.

Anderson Cancer Center, USA and Dr. Harald Paganetti from Massachusetts General Hospital, USA for their collaboration on this research.

\section{References}

1) R. Wilson, "Radiological use of fast protons," Radiology, 47, 487-491 (1946).

2) H. Paganetti, "Four-dimensional Monte Carlo simulation of time-dependent geometries," Phys. Med. Biol., 49[6], N75-N81 (2004).

3) S. Agostinelli et al, "GEANT4 - a simulation toolkit," Nucl. Instr. Meth. Phys. Res., A506[3], 250-303 (2003).

4) D. Pelowitz, (editor), MCNPX ${ }^{T M}$ user's manual, version 2.6.0, LA-CP-07-1473, Los Alamos National Laboratory (LANL) (2008). 
5) U. Titt, N. Sahoo, X. Ding, Y. Zheng, D. Newhauser, R. Zhu, C. Polf, T. Gillin,d R. Mohan, "Assessment of the accuracy of an MCNPX-based Monte Carlo simulation model for predicting three-dimensional absorbed dose distributions," Phys. Med. Biol., 53[16], 4455-4470 (2008).

6) W. Newhauser, J. Fontenot, Y. Zheng, J. Polf, U. Titt, N. Koch, $\mathrm{X}$. Zhang, R. Mohan, "Monte Carlo simulations for configuring and testing an analytical proton dose-calculation algorithm," Phys. Med. Biol., 52 [15], 4569-4584 (2007).

7) B. Gottschalk, NEU User Guide, Harvard University, Cambridge, MA, USA, (2006).

8) W. Chu, B. Ludewigt, T. Renner, "Instrumentation for treatment of cancer using proton and light-ion beams," Rev. Sci. Instrum., 64[8], 2055-2122 (1993).

9) Y. Zheng, J. Fontenot, P. Taddei, D. Mirkovic and W. Newhauser, "Monte Carlo simulations of neutron spectral fluence, radiation weighting factor and ambient dose equivalent for a passively scattered proton therapy unit," Phys. Med. Biol.,
53[1], 187-201 (2008).

10) ICRU, Prescribing, recording, and reporting proton-beam therapy: ICRU Report, No. 78, International Commission on Radiation Units and Measurements (2007).

11) V. Vavilov, "Ionization losses of high-energy heavy particles," Sov. Phys. Jetp-Ussr., 5, 749-751 (1957).

12) S. Mashnik, A. Sierk, K. Gudima, M. Baznat, "CEM03 and LAQGSM03 - new modeling tools for nuclear applications," $J$. Phys. Conf. Ser., 41, 340-351 (2006).

13) X-5 Monte Carlo Team, MCNP-a general Monte Carlo $N$-particle transport code, version 5, LA-UR-03-1987, Los Alamos National Laboratory (LANL) (2003).

14) F. Brown, J. Sweezy, R. Hayes, Monte Carlo parameter studies and uncertainty analyses with MCNP5, LA-UR-04-0499, Los Alamos National Laboratory (LANL) (2004).

15) F. Brown, A tutorial on merging tallies from separate MCNP5 runs, LA-UR-08-0249, Los Alamos National Laboratory (LANL) (2008). 